\title{
Filtering Normal Retinal Images for Diabetic Retinopathy Screening Using Multiple Classifiers
}

\author{
Jonathan Goh, Lilian Tang, George Saleh, Lutfiah Al turk, Yu Fu, Antony Browne
}

\begin{abstract}
Diabetic retinopathy is a complication of diabetes and early detection is essential for effective treatment. In this paper, a novel technique for the separation of normal and abnormal retinal images is described. Various features are extracted from local sub images and then fed through multiple classifiers to categorise them into interim classes followed by a reasoning process to give a more reliable and robust result. This is then followed by a global analysis to decide the normality of the whole image.
\end{abstract}

Index Terms - Diabetic Retinopathy Screening, Multiple Classifiers, Ensembles, Pattern Recognition

\section{INTRODUCTION}

Diabetic retinopathy (DR) is an eye disease that has been one of the major causes of blindness in the world for adults under the age of 65 [1]. Early detection of DR through screening can prevent blindness and allow for maintenance of good vision. A typical screening process involves the acquisition of retinal images from the patient followed by manual examination of each individual image by medical experts (these are actually often technicians trained by medics) in order to identify any signs of deterioration. This process is known to be inefficient, expensive and time consuming.

These few reasons alone make the development of an automated system imperative. Previous work has been focusing on detecting clinical sighs (in abnormal images) such as haemorrhages, exudates $[2,3,4,5,6]$ and micro aneurysms [7, 8] that exhibit as DR progresses. In current clinical practice, experts usually spend a much longer time to decide whether an image is indeed really normal to minimise false positive, whilst judging an image with true abnormality is much quicker and easier. The motivation of this work is to develop a system that is able to distinguish retinal images with and without any DR signs in order to accurately filter out normal images, so that medical experts can focus solely

Manuscript received June 30, 2009.

Jonathan Goh is with the Department of Computing, University of Surrey, Guildford, Surrey, GU2 7XH, United Kingdom (Phone: +44 7525754397; email: jg00004@surrey.ac.uk).

Lilian Tang is with the Department of Computing, University of Surrey, Guildford, Surrey, GU2 7XH, United Kingdom (e-mail: htang@surrey.ac.uk).

George Saleh is with Moorfields Eye Hospital NHS Foundation Trust, 162 City Road, London, EC1V 2PD, United Kingdom.

Lutfiah Al turk is with the Department of Statistics, King Abdulaziz University, Kingdom of Saudi Arabia.

$\mathrm{Yu} \mathrm{Fu}$ and Antony Browne, were both with the Department of Computing, University of Surrey, Guildford, Surrey, GU2 7XH, United Kingdom. on the abnormal cases, saving screening time, manpower and cost.

In order to distinguish normal and abnormal retina images, different techniques must be employed for the system to understand the presence of different clinical signs. In the literature, image processing techniques such as dynamic thresholding [2, 3] and matched filters [4] have been applied to detect exudates. However, these techniques do not contain any recognition models that are able to distinguish between visually similar symptoms. Classification algorithms have also been utilised in $[5,6]$ to distinguish between exudates and cotton wool spots, but the single classifier used is unable to maintain accuracy over a large volume of diverse images. Also, many retina images are visually very similar and usually defer with very subtle details. However, these subtle differences are often of great clinical importance. The automated system must be able to identify such subtlety while ensuring consistent accuracy across a diverse data set.

In our work, an algorithm has been developed to separate normal and abnormal retina images. The first stage aims at dividing the image into smaller sub images, followed by feature extraction. Due to uneven illumination, the image is firstly partitioned into 32 by 32 pixel sub images to minimise this effect. In the second stage, multiple classifiers are used to categorise the sub images into interim classes. A reasoning process is then carried out to determine the abnormality of the image. The work has been tested over a large-scale database taken from various sources.

This paper is organised as follows. In Section II, we present the techniques employed used in the proposed algorithm. In Section III, experimental results are given. Finally, we summarise this paper in Section IV.

\section{PROPOSED FRAMEWORK}

In this section, we describe the proposed framework to extract clinical signs to determine whether an image is normal or abnormal. One of the main obstacles is the variability in the retinal image, depending on factors such as degree of pigmentation of epithelium and choroid in the eye, size of pupil, illumination, disease, imaging settings (which can vary even with same equipment), patients' ethnic origin, and other variants. These factors, affect the appearance of various fundus features. Another challenge in detecting clinical signs is their similarity to other anatomical structures. For example, exudates and cotton wool spots are similar in colour to the optic disc; haemorrhages and micro aneurysms are similar to blood vessels, posing great ambiguity that requires fine analysis through context. All aspects of a 
recognition process of DR images of such feature representation, classification and contextual analysis cannot be treated in isolation. Therefore, an integral approach has been proposed.

Figure 1 shows the design of the proposed framework. Details of each step of the algorithm are presented in this section. The image is first divided into 32 by 32 pixels sub images to reduce the effects of uneven illumination. Nine different sets of features are then extracted from each of these sub images and fed into the nine different ensembles. The results from the ensembles are combined to obtain the most likely label for the sub image. An obvious abnormal image tends to have many sub images that are detected as clinical signs as compared to a real normal image or an abnormal image which displays very subtle clinical signs. For a normal image, the detector has to be sure whether it is a misclassification or an image with subtle symptom. Therefore, a reasoning process is carried out on images with less than five sub images identified as abnormal signs to ensure the final output of these sub images are indeed certain.

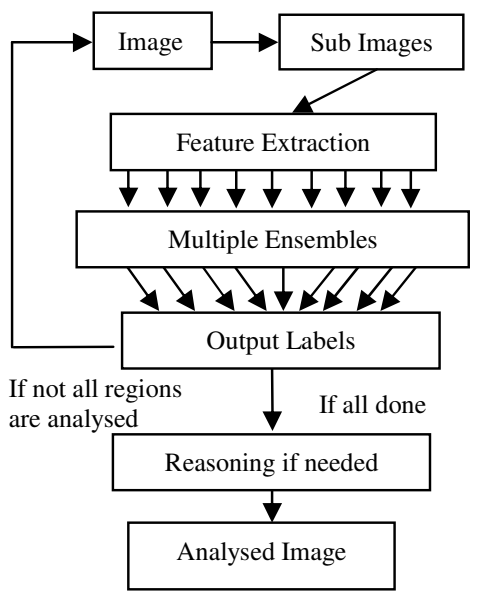

Figure 1 Proposed Framework

\section{A. Multiple Classifiers}

Traditionally, to find the best classifier for a single problem, a few classification schemes will be developed using different sets of features, training algorithms, etc. followed by experimentation and evaluation of the application to determine the best classifier. However, while each of these classifiers may be successful to a certain extent, neither of them would be a perfect solution to a given problem.

It has been reported by Kittler et al [9] that although the best classifier would give the best performance, samples that are misclassified by the best classifier would not necessarily be misclassified by the rest of the classifiers. The combination of different classifiers may potentially offer information that can be used to improve the performance of the best classifier.

In our work, various features are used to represent the same pattern and a hierarchical structure has been developed as the classification strategy as shown in figure 2 . The median rule is used in the first layer and the maximum rule for the second layer to derive the final output.

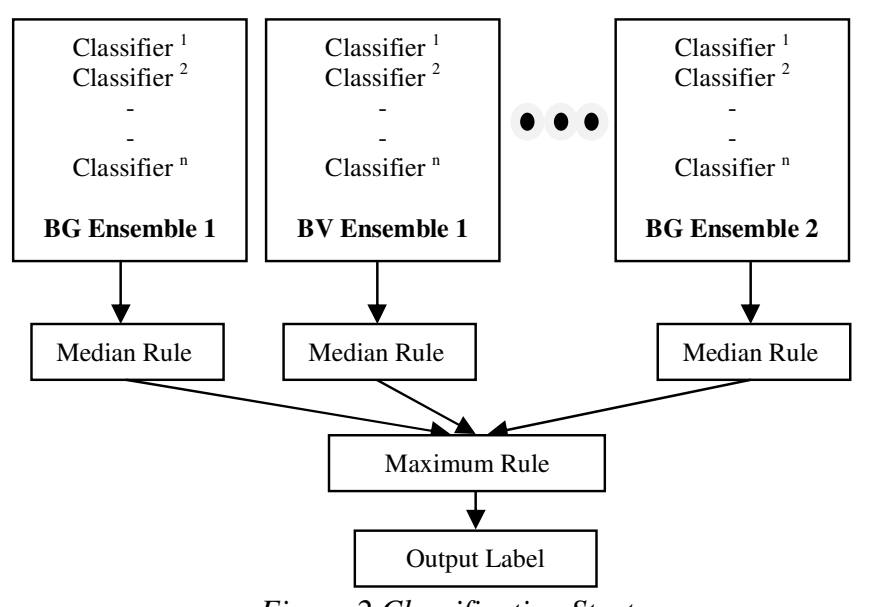

Figure 2 Classification Strategy

Ensembles of Multi Layer Perceptron networks are trained using the model developed in [10]. Each ensemble is trained using different training algorithms (Scaled Conjugate Gradient algorithm, Quasi Newton algorithm and the Conjugate algorithm), various hidden units (multiples of 2 ranging from 2 to 20) and different initial weights are randomly generated from different ranges (-0.0 to 0.01, 0.001 to 0.001 and -0.0001 to 0.0001 ) resulting in a total of 90 classifiers for each ensemble. The results from each of the individual classifiers are combined using the median rule as the output of the ensemble.

Nine ensembles of neural networks are trained using totally different features to categorise between different classes. Three ensembles have been developed and trained to detect background sub images (BG), four ensembles to detect blood vessels (BV) and two ensembles to detect bright lesions.

\section{B. Feature Extraction}

\section{i. $\quad$ Colour Information}

One of the features we used to design the base classifier is colour information of the sub image. This information can be generated through the histogram. Using this feature, we developed the background classifier and the blood vessel classifier to identify background sub images and blood vessel sub images.

\section{ii. $\quad$ Fourier Spectra}

It is observed that there is a distinct peak in the Fourier spectra if an object is present in the sub image compared with just a pure background sub image which often has a smooth descent in the Fourier spectra. Thus, the Fourier spectra have been used as a feature to develop another background base classifier to classify between background and nonbackground sub images. 


\section{iii. Principal Component Analysis (PCA)}

Principal Component Analysis (PCA) has been primarily used to reduce the size of complex data and it has been used widely in many applications [11, 12]. In our work, as the RGB image is far too large to be used as an input feature, we used this technique to reduce the size of the 32 by 32 RGB sub image to reduce the dimension of the input space. The first 15 components are used as features for the third background classifier and the first 20 components are as features for the blood vessels classifiers respectively.

\section{iv. $\quad$ Phase symmetry}

Blood vessels have this unique property that they gradually grow out from the optic disc. If these blood vessels are segmented into smaller segments, they will most certainly exhibit a linear characteristic.

Therefore, a phase symmetry [13] line feature detector has been applied to determine whether any line exists in the shade corrected sub image as the blood vessels appear more prominent in them. Subsequently, image-processing techniques are used to detect the full region of the blood vessels before extracting useful features such as mean colour values, mean shade corrected values, length and size to develop a blood vessel base classifier.

A phase symmetry [13] blob detector has also been used to detect bright clinical signs in the sub images. The response generated by the blob detector is then dimensionally reduced using PCA. Through experimentation, the top 20 principal components are used as input feature for the bright lesion classifier.

\section{v. $\quad$ Texture Analysis}

Gabor filters has been used previously in many image analysis applications for texture analysis and segmentation $[14,15]$. For the background classifier, the extracted Gabor features are the mean and variance of the difference responses generated by the four different filters of different orientations.

However, for the blood vessel classifier, twelve filters of different orientations are used to ensure that maximum responses are obtained since blood vessels can appear in the sub images with unpredictable orientations.

Statistical texture analysis using properties such as contrast levels and the homogeneity of the image is also carried out to develop a bright lesion classifier.

\section{Contextual Reasoning}

On reviewing the results from the initial experiments as well as the output of the ensembles, it is observed that there are more than one detector that obtain an output with high probability suggesting that there may be more than one object in the sub image containing either a blood vessel, a bright lesion or both. These can easily lead to misclassification as what actually have happened.

The reasoning processing is based on the properties of these bright lesions. Although exudates can appear as a small bright yellow object in the image, this is usually very rare as they usually appear in patches of various sizes, thus may fill more than one sub image. Therefore, in our reasoning process, the neighbourhoods of the sub image at focus will be scanned for any other signs of bright lesions. If they do exist, the probability of the classified sub image will be enforced to be a correct classification.

If no neighbourhoods are detected as bright lesions, the reasoning module will refer to the next most likely possibility of the sub image which can either be a blood vessel or a background if the probabilities are above a certain threshold. The reasoning module will then look up the confusion matrix to further confirm its belief.

The confusion matrix is created based on the testing data and is used to determine the performance of the classifiers as well as which classes are often misclassified as others. For example, the confusion matrix shows that the exudates class is often misclassified as a blood vessel class i.e., if a sub image is suspected as non-exudates, there is a high likelihood that it might contain blood vessels.

By using this information, the next most probable label from the classification process for the sub image will then be compared with the data generated by the confusion matrix. If the next likely label is coherent with the data from the confusion matrix, then the label will be changed to the latter.

However, if none of the background or blood vessel detectors are above the threshold of 0.5 , it is very likely that the clinical sign is too subtle for the classifiers to detect using their textural properties. Hence, another classifier which utilises other visual properties is used to further identify the sub image as a bright lesion.

\section{EXPERIMENTAL RESULTS}

In order to determine the effectiveness of our proposed technique, 1000 retina images obtained from various sources including the Optimal Detection and Decision-Support Diagnosis of Diabetic Retinopathy database (DIARETDBO, DIARETDB1) [16] are used to test the system. By using the data from various sources, this ensures that the data are more diverse, hence, testing the system to its full potential. As the optic disc has visually similar characteristics to those of bright lesions, an optic disc localisation algorithm [17] is first used to automatically mark out the location so as not to be detected as false positives.

\section{A. Separating Normal and Abnormal Images.}

Using the ensembles as the main classifier, the application managed to successfully detect 685 out of 750 abnormal images giving an accuracy of $91 \%$. On detecting normal images, the application successfully detected 230 out of 250 normal images giving an accuracy of $92 \%$.

While using multiple classifiers can effectively deal with diversity, the hierarchical multiple classifier combination strategy also provides excellent tolerance to classification errors. However, misclassifications still occur. As mentioned earlier, initial experimental results showed that there are certain sub images which are often misclassified due to their visual similarities. Hence, a reasoning process was needed to re-label such misclassifications using the information generated by the confusion matrix. Through the reasoning 
process, we managed to lower the false positive of detecting normal images by $11 \%$. One of the significant issues was the amount of false positives due to the bright lesion appearing very subtle near the vicinity of the blood vessels. In this situation, the output from the blood vessel ensemble would be higher than the bright lesion ensemble, resulting in the sub image being labelled as a blood vessel. Currently, the reasoning process is triggered only based on the number of detected bright lesion if they are less than 5 sub images; our ongoing work is investigating how reasoning could be included on all images to deal with these situations for a more accurate analysis.

The actual system output with labels is in figure 3 (BV blood vessel (the system labels such areas as "BV" in blue letters), BG - background (in green), OD - optic disc (in white), EX - exudates (in yellow)).

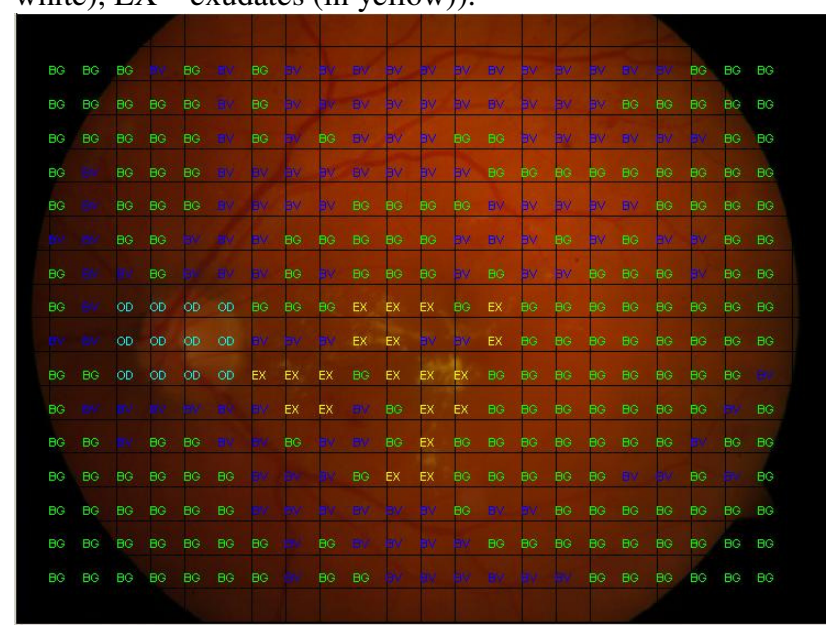

Fig. 3 Actual System Output - an image with Classified Labels

\section{B. Sensitivity and Specificity}

For the second part of the experiment, we further analyse the extent of the accuracy using ensembles to separate normal and abnormal images and determine their sensitivity of 0.91 and specificity of 0.92 using the following equations.

$$
\text { Sensitivity }=\frac{\text { TruePositive }}{\text { TruePositive }+ \text { FalseNegatives }}
$$

$$
\text { Specificity }=\frac{\text { TrueNegative }}{\text { TrueNegative }+ \text { FalsePositives }}
$$

\section{CONCLUSIONS}

In summary, we have demonstrated a novel way in separating normal and abnormal retinal images using multiple classifiers. The experimental results have shown that by using multiple classifiers we can achieve an accuracy of $92 \%$ in detecting normal images and $91 \%$ in detecting abnormal images over 1000 retina images. We have also demonstrated that the reasoning process can correct the misclassified sub images to produce a more robust application. Although in this paper we only discuss the system that is currently only detecting abnormal images with bright lesions while deciding the visual characteristics of normal images, our ongoing work includes detection of other dark lesion such as micro aneurysms and haemorrhages. Also, a global analysis would be carried out through reasoning on all sub images for a more accurate diagnosis.

\section{REFERENCES}

[1] World Health Organisation. [Internet], <Available from http://www.who.int/blindness/causes/priority/en/index6.html/> Last Access [02/03/08]

[2] Zheng Liu, Chutatape Opas, Shankar M Krishnan, (1997) “Automatic image analysis of fundus photograph", Proceedings of the 19th International Conference - IEEE/EBMS, Chicago, IL, USA. H. Poor, An Introduction to Signal Detection and Estimation. New York: Springer-Verlag, 1985, ch. 4.

[3] Anantha Vidya Sagar, S. Balasubramaniam, V. Chandrasekaran, (2007) "A novel integrated approach using dynamic thresholding and edge detection for automatic detection of exudates in digital fundus retinal images", Proceedings of the International Conference on Computing: Theory and Application.

[4] Huiqi Li, Opas Chutatape, (2000) "Fundus Image Features Extraction", IEEE 22nd Annual EMBS International Conference, Chicago, IL, USA.

[5] A. Osareh, M. Mirmehdi, B. Thomas, R. Markham, (2003) "Automated Identification of Diabetic Retinal Exudates in Digital Color Images", British Journal of Ophthalmology, pp1220-1223.

[6] Zhang Xiaohui, Opas Chutatape, (2004) "Detection and Classification of Bright Lesions in Color Fundus Images", IEEE International Conference on Image Processing. Singapore.

[7] Allan J. Frame, Peter E. Undrill, Micheal J. Cree, John A. Olson, Kenneth C. McHardy, Peter F. Sharp, John V. Forrester, (1998) "A comparison of computer based classifcation methods applied to the detection of microaneurysms in ophthalmic fluorescein angiograms", Computers in Biology and Medicine, Vol. 28(3), pp225-238.

[8] A.M. Mendonca, A. J. Camilho, J.M. Nunes, (1999) "Automatic Segmentation of Microaneurysms in Retinal Angiograms of Diabetic Patients", 10th International Conference on Image Analysis and Processing (ICIAP'99), Veneza, Itália.

[9] Joseph Kittler, Mohamad Hatef, Robert P.W. Duin and Jiri Matas, (1998) "On Combining Classifiers", IEEE Transactions on Pattern Analysis and Machine Intelligence. Vol.3(3).

[10] Y. Fu and A. Browne, (2007), "Using Ensembles of Neural Networks to Improve Automatic Relevance Determination", Proceedings of International Joint Conference on Neural Networks, Orlando, Florida, USA.

[11] Turk, M., Pentland, A. (1991), "Eigenfaces for recognition", The Journal of Cognitive Neuroscience, 3(1): 71-86.

[12] Zhang Xiaohui, Opas Chutatape, (2005), "Top-down and Bottom-up Strategies in Lesion Detection of Background Diabetic Retinopathy", IEEE Computer Society Conference on Computer Vision and Pattern Recognition, 2005.

[13] Peter Kovesi (2003), "Phase Congruency Detects Corners and Edges" The Australian Pattern Recognition Society Conference: DICTA 2003. December 2003. Sydney. pp 309-318.

[14] Vibha S. Vyas, Priti Rege (2006), "Automated Texture Analysis with Gabor Filter", Graphics, Vision, and Image Processing Journal, Vol. 6 , Issue 1.

[15] Tati Rajab Mengko, J. Tjandra Pramudito (2002), "Implementation of Gabor Filter to Texture Analysis of Radiographs in the Assessmen of Osteoporosis", Asia-Pacific Conference on Circuits and Systems.

[16] Optimal Detection and Decision-Support Diagnosis of Diabetic Retinopathy (2005) [Internet]<Available from http://www.it.lut.fi/project/imageret/> Last accessed [03/03/08]

[17] Osareh A, Mirmehdi M, Thomas, B (2002), "Comparison of Colour Spaces for optic disc localisation in retinal images", $16^{\text {th }}$ International Conference on Pattern Recognition. 